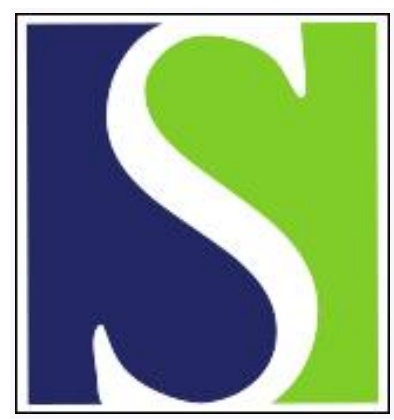

Scand J Work Environ Health 2011;37(3):196-203

https://doi.org/10.5271/sjweh.3136

Published online: 25 Nov 2010, Issue date: May 2011

Kettlebell training for musculoskeletal and cardiovascular health: a randomized controlled trial

by Jay K, Frisch D, Hansen K, Zebis MK, Andersen CH, Mortensen OS, Andersen LL

Affiliation: National Research Centre for the Working Environment, DK-2100 Copenhagen Ø, Denmark. Ila@arbejdsmiljoforskning.dk

Refers to the following text of the Journal: 2004;30(5):390-398

The following article refers to this text: 2015;41(2):153-163

Key terms: ballistic training; cardiovascular health; fitness; intervention; kettlebell training; MSD; muscle strength; musculoskeletal disorder; musculoskeletal health; neck pain; randomized controlled trial; workplace

This article in PubMed: www.ncbi.nlm.nih.gov/pubmed/21107513 


\title{
Kettlebell training for musculoskeletal and cardiovascular health: a randomized controlled trial
}

\author{
by Kenneth Jay, BSc, ${ }^{1}$ Dennis Frisch, BSc, ${ }^{1}$ Klaus Hansen, BSc, ${ }^{1}$ Mette K Zebis, PhD, ${ }^{3}$ Christoffer H \\ Andersen, MSc, ${ }^{1}$ Ole S Mortensen, PhD, ${ }^{1,2}$ Lars L Andersen, PhD ${ }^{1}$
}

\begin{abstract}
Jay K, Frisch D, Hansen K, Zebis MK, Andersen CH, Mortensen OS, Andersen LL. Kettlebell training for musculoskeletal and cardiovascular health: a randomized controlled trial. Scand J Work Environ Health. 2011;37(3):196-203. doi:10.5271/sjweh.3136
\end{abstract}

Objective The aim of this trial was to investigate the effectiveness of a worksite intervention using kettlebell training to improve musculoskeletal and cardiovascular health.

Methods This single-blind randomized controlled trial involved 40 adults from occupations with a high prevalence of reported musculoskeletal pain symptoms (mean age 44 years, body mass index $23 \mathrm{~kg} / \mathrm{m}^{2}, 85 \%$ women, with pain intensity of the neck/shoulders 3.5 and of the low back 2.8 on a scale of $0-10$ ). A blinded assessor took measures at baseline and follow-up. Participants were randomly assigned to training - consisting of ballistic fullbody kettlebell exercise 3 times per week for 8 weeks - or a control group. The main outcome measures were pain intensity of the neck/shoulders and low back, isometric muscle strength, and aerobic fitness.

Results Compared with the control group, pain intensity of the neck/shoulders decreased 2.1 points [95\% confidence interval $(95 \% \mathrm{CI})-3.7-0.4]$ and pain intensity of the low back decreased 1.4 points $(95 \% \mathrm{CI}-2.7-$ -0.02 ) in the training group. Compared with the control group, the training group increased muscle strength of the trunk extensors $(\mathrm{P}<0.001)$, but not of the trunk flexors and shoulders. Aerobic fitness remained unchanged.

Conclusions Worksite intervention using kettlebell training reduces pain in the neck/shoulders and low back and improves muscle strength of the low back among adults from occupations with a high prevalence of reported musculoskeletal pain symptoms. This type of training does not appear to improve aerobic fitness.

Key terms ballistic training; fitness; intervention; muscle strength; musculoskeletal disorder; MSD; neck pain; workplace.

Musculoskeletal disorders are common and costly. In a survey of 29828 representative US adults, Strine \& Hootman (1) reported that the three-month prevalence of back and/or neck pain was $31 \%$. Linton (2) reported a widespread prevalence of back pain in a representative sample of 22180 adult Scandinavian workers. In that study, 39\% had experienced back pain during the previous year, and $16 \%$ had sought out medical treatment for their pain. Furthermore, longitudinal observations from the UK and Sweden show that low-back pain is the predominant cause of absence from work with more than $12.5 \%$ and $13.5 \%$ of all annual sick days, respectively (3). Low-back pain therefore represents a major socioeconomic burden.
The prevalence of neck and shoulder pain is also extensive. In the working population, it is estimated that $20-30 \%$ have symptoms of musculoskeletal dysfunction in the upper extremity (4). The cross-sectional study by Linton (2) reported that $31 \%$ of the population experienced neck pain and $18 \%$ had seen a medical healthcare professional for such pain during the previous year. In a Canadian survey, the prevalence of neck pain in the general population was $>50 \%$ over the course of six months (5). Neck and shoulder pain is especially prevalent among sedentary workers in occupations characterized by frequent repetitive motions (eg, lab technicians and office workers) (6). Neck/shoulder pain is also a risk factor for long-term sickness absence among white-collar workers (7)

1 National Research Centre for the Working Environment, Copenhagen, Denmark.

2 Department of Occupational and Environmental Medicine, Bispebjerg University Hospital, Copenhagen, Denmark.

3 Gait Analysis Laboratory, Hvidovre University Hospital, Hvidovre, Denmark.

Correspondence to: Lars L Andersen, National Research Centre for the Working Environment, Lersø Parkalle 105, DK-2100 Copenhagen Ø, Denmark. [E-mail: 1la@arbejdsmiljoforskning.dk] 
Physical exercise has been found to be an effective intervention for pain and discomfort in the neck and low-back area $(8,9)$, but the evidence for or against a specific type of exercise is inconclusive (9). Some investigations show that specific training to improve muscle strength has a significant and prolonged positive effect on neck pain $(10,11)$, while others report effectiveness of both all-round exercise and specific strength training for low-back and neck pain $(12,13)$. Further, aerobic training such as running and cycling improves fitness and lowers cardiovascular risk factors (14).

Although training for both muscle strength and aerobic fitness is essential for overall health, few people have the time to do several different types of exercise. Recent trends in physical fitness and performance training suggest various alternatives to the already known and established methods for improving both aerobic fitness and muscle strength - kettlebell training being one of them. In short, a kettlebell is a cannonball-shaped iron orb with a handle attached to it. With an offset center of gravity, the kettlebell is ideally suited for ballistic full-body exercise involving high muscle forces, and thereby a potential candidate for improving both muscle strength and aerobic fitness. A recent study by Farrar and coworkers (15) indicated that a typical intermittent kettlebell-training protocol poses a metabolic challenge of sufficient intensity to create cardiovascular adaptations, but no randomized controlled trials have confirmed this.

Our study investigates - in a randomized controlled trial - the effect of kettlebell training on neck/shoulder and low-back pain, muscle strength, and aerobic fitness in a group of adults working in occupations with a high prevalence of reported musculoskeletal pain symptoms. We hypothesize that kettlebell training reduces musculoskeletal pain symptoms and improves muscle strength and fitness.

\section{Methods}

\section{Study design and flow of participants}

We performed a randomized controlled trial in Copenhagen, Denmark, from March-May 2010. Figure 1 shows the flow of participants through the study. We aimed to recruit 50 eligible participants for the study. A screening questionnaire went out to 174 employees - mainly laboratory technicians - in a large pharmaceutical company, and 90 replied. The inclusion criterion was willingness to participate in the study (yes/no on the screening questionnaire); a defined level of musculoskeletal pain was not required. Predefined exclusion criteria, which lead to the exclusion of 12 employees, were: (i) a medical history of life threatening disease
$(\mathrm{N}=0)$, (ii) traumatic injury of the neck or back $(\mathrm{N}=5)$, (iii) other serious chronic disease ( $\mathrm{N}=2$ ), or (iv) pregnancy $(\mathrm{N}=5)$. Furthermore, 21 employees replied to the questionnaire, but declined participation in the study. We invited the remaining 57 employees for a physical examination, and 43 showed up. During the physical examination, we excluded three employees who had blood pressure above 160/100. The examiner measured muscle strength and aerobic fitness of the remaining 40 participants as described hereafter. Table 1 shows baseline demographics and physical characteristics of the 40 included participants. At baseline, there were no significant differences between the two groups.

The Local Ethical Committee approved the study protocol (HC2008103). All participants gave written informed consent in agreement with the Declaration of Helsinki. We registered the study prior to enrolment of participants (www.clinicaltrials.gov, number NCT01076127).

Using a computer-generated random numbers table, we randomly allocated the 40 participants to the training or control group following the baseline examination of all participants. We kept the examiner blinded, and instructed the participants not to reveal their particular intervention during the follow-up examination.

\section{Musculoskeletal pain}

We asked the participants to rate their pain at baseline and follow-up via an internet-based questionnaire. Participants rated their average neck/shoulder and low back pain during the previous month on a visual-analog scale from $0-10$, where 0 is "no pain" and 10 is "the worst imaginable pain" (16). The neck/shoulder area and low back area was defined with a drawing from the Nordic Questionnaire (17).

\section{Muscle strength}

Using a standardized procedure, the examiner measured the participants muscle strength of the low back, trunk, and shoulders (18). Participants performed maximal voluntary contractions (MVC) during static trunk flexion, back extension, and shoulder elevation. For the shoulder measurement, the participant was sitting upright in a height-adjustable chair, and for the other measurements the participant was standing upright. We used Bofors dynamometers (Bofors Elektronik, Karlskoga, Sweden) to measure force, which was converted to torque by multiplying the force with the individual lever arm length. The examiner instructed the participant to gradually build up the force over 5 seconds, then to keep the maximal force for 2-3 seconds, and, finally, slowly to lower the force to zero. For each exercise, participants performed at least three MVC separated by rest periods 


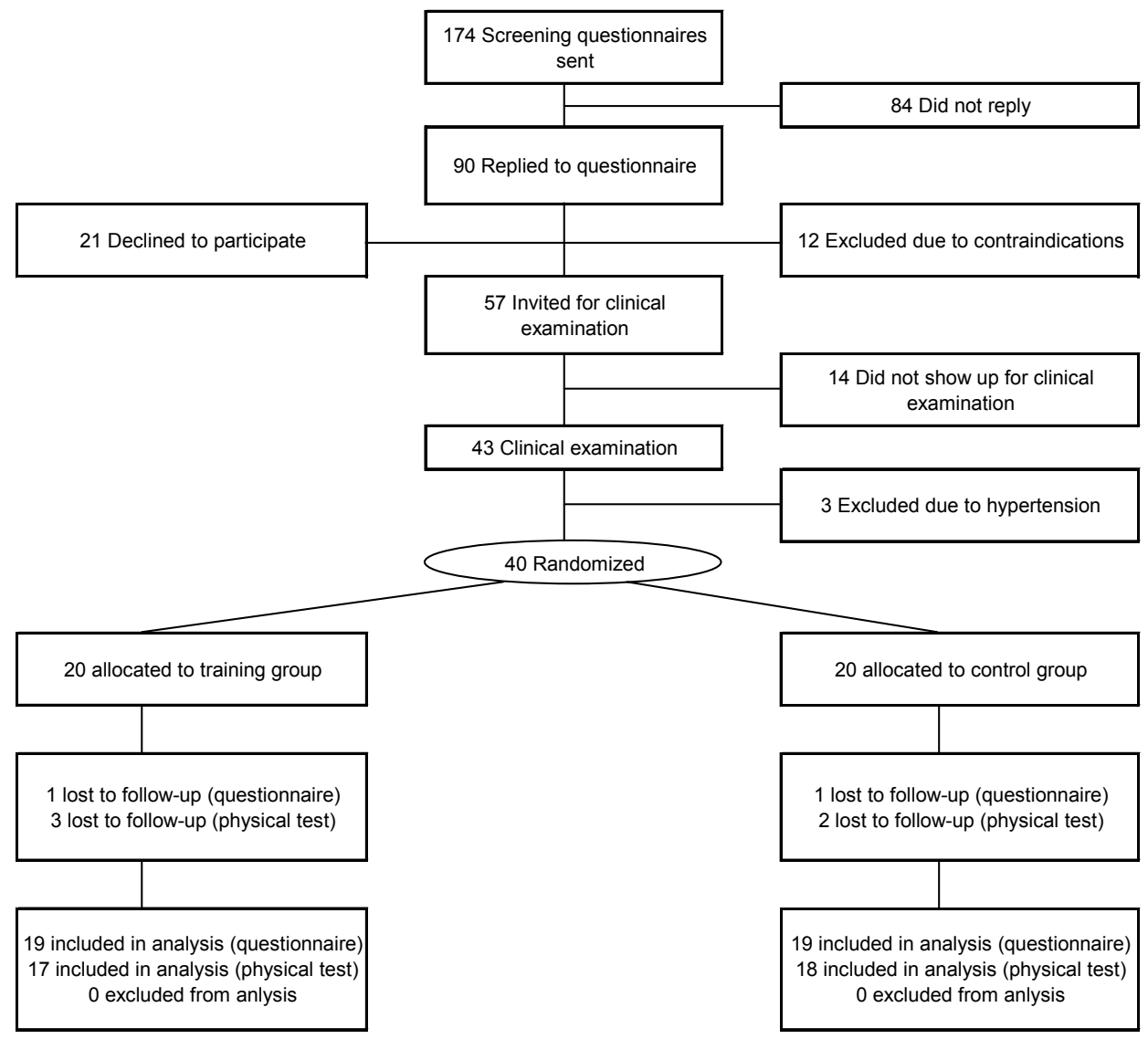

Figure 1. Flow of participants through the study.

Table 1. Baseline demographics and physiological characteristics of the training $(\mathrm{N}=20)$ and control group $(\mathrm{N}=20)$. [SD=standard deviation; $\mathrm{MVC}=$ maximal voluntary contraction (ie, muscle strength); $\mathrm{VO}_{2} \mathrm{Max}=$ maximal oxygen uptake.]

\begin{tabular}{|c|c|c|c|c|}
\hline & \multicolumn{2}{|c|}{ Training a } & \multicolumn{2}{|c|}{ Control a } \\
\hline & Mean & SD & Mean & SD \\
\hline \multicolumn{5}{|l|}{ Demographics } \\
\hline Age (years) & 44 & 8 & 43 & 10 \\
\hline Height $(\mathrm{cm})$ & 169 & 7 & 172 & 9 \\
\hline Weight (kg) & 68 & 11 & 66 & 11 \\
\hline Body mass index $\left(\mathrm{kg} / \mathrm{m}^{2}\right)$ & 24 & 3 & 22 & 2 \\
\hline \multicolumn{5}{|l|}{ Musculoskeletal } \\
\hline Intensity of low-back pain $(0-10)$ & 2.8 & 3.0 & 2.7 & 2.0 \\
\hline Intensity of neck/shoulder pain $(0-10)$ & 3.7 & 1.8 & 3.2 & 1.7 \\
\hline MVC - back extension (Nm) & 136 & 42 & 145 & 37 \\
\hline MVC - trunk flexion (Nm) & 120 & 42 & 126 & 41 \\
\hline MVC - shoulder elevation (Nm) & 63 & 18 & 62 & 25 \\
\hline \multicolumn{5}{|l|}{ Cardiovascular } \\
\hline Aerobic fitness $\left(\mathrm{VO}_{2} \mathrm{Max}: \mathrm{ml} \mathrm{O}_{2} / \mathrm{kg} / \mathrm{min}\right)$ & 37 & 9 & 39 & 8 \\
\hline Systolic blood pressure $(\mathrm{mmHg})$ & 116 & 30 & 118 & 11 \\
\hline Diastolic blood pressure $(\mathrm{mmHg})$ & 81 & 8 & 75 & 7 \\
\hline \multicolumn{5}{|l|}{ Work-related } \\
\hline Computer use (percentage of worktime) & 61 & 17 & 64 & 17 \\
\hline Weekly working hours & 39 & 3 & 40 & 3 \\
\hline \multicolumn{5}{|l|}{ Leisure-time physical activity (hours/week) } \\
\hline Light & 3.0 & 1.8 & 3.1 & 1.6 \\
\hline Moderate & 2.1 & 1.4 & 2.3 & 1.4 \\
\hline Vigorous & 0.2 & 0.4 & 0.2 & 0.4 \\
\hline
\end{tabular}

a Number of women $=17$, number of men $=3$ 


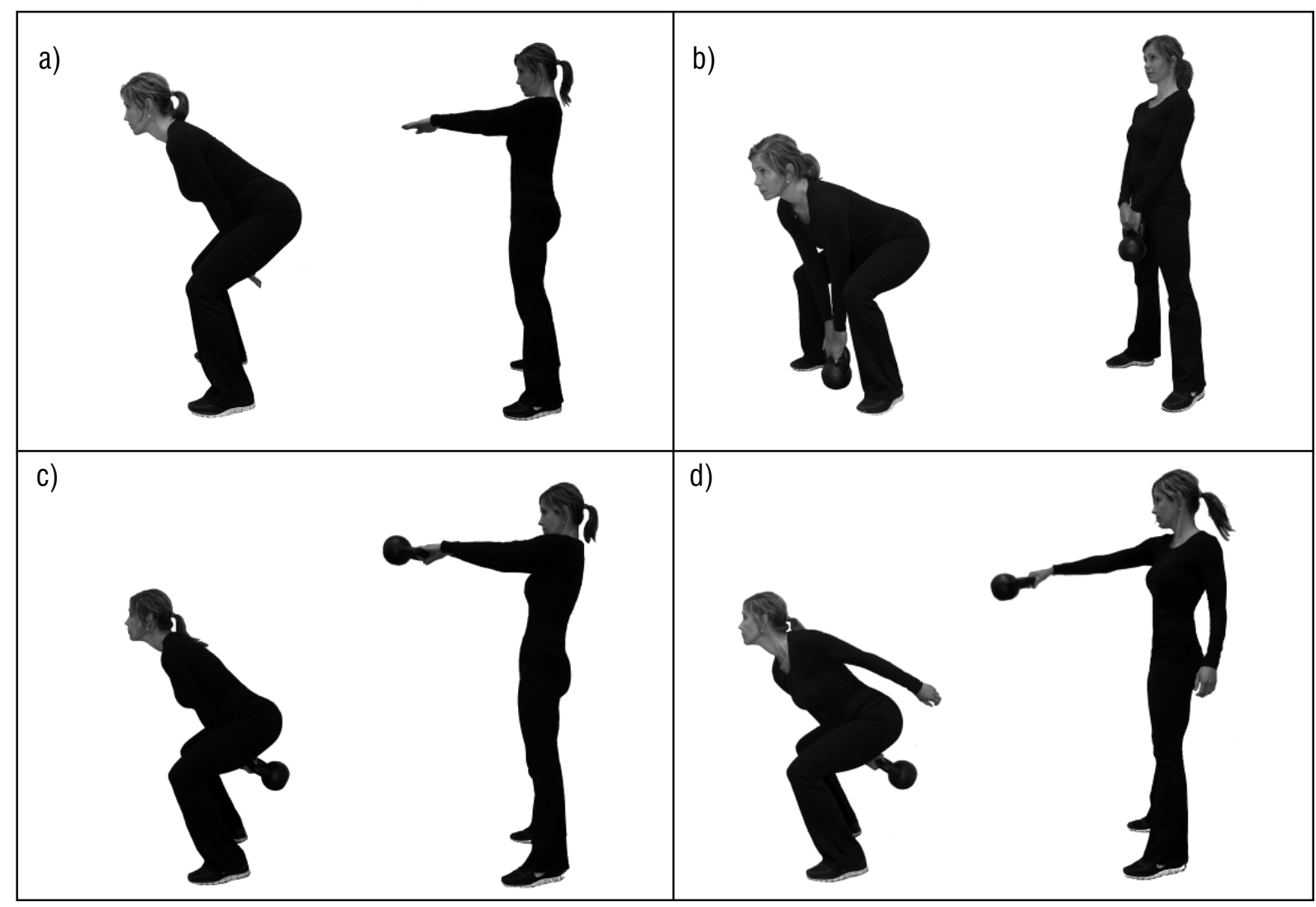

Figure 2. Illustration of the four progression levels used during the training sessions: (a) the unweighted swing, (b) deadlift with a kettlebell, (c) two-handed swing with a kettlebell, and (d) one-handed swing with a kettlebell.

of one minute. Participants performed an extra MVC if they improved their $3^{\text {rd }}$ MVC by more than $5 \%$ of the previous best attempt. The same procedure applied for the $4^{\text {th }}$ MVC, for a maximal number of five MVC.

\section{Aerobic fitness}

We used Åstrand's standardized method to estimate aerobic fitness (maximal oxygen uptake: $\mathrm{VO}_{2} \mathrm{Max}$ ) during a submaximal workload provided by a stationary Monark cycle ergometer, model Ergomedic 874E (Monark AB, Stockholm, Sweden) (19).

\section{Intervention}

Training was performed 3 days per week for 8 weeks. Lasting 20 minutes, each session included a 5-10 minute warm up and a 10-15 minute interval training. The warm up consisted of dynamic stretches and practicing exercise-specific movements and positions. The interval training consisted of 10 intervals of 30 seconds with rest period of 30-60 seconds. The intervals consisted of constant work with participants choosing an appropriate progression level as follows: (i) unweighted swing (figure 2a); (ii) deadlift with a kettlebell (figure 2b); (iii) two-handed swing with a kettlebell (figure 2c); and (iv) one-handed swing with a kettlebell (figure 2d).

During the first 4 weeks of training, the rest between sets was one minute of active recovery. During the last 4 weeks, the rest was compressed to 30 seconds of active recovery. The active recovery consisted of walking and drills focusing on shaking arms and legs. An experienced kettlebell instructor supervised all training.

All participants started learning the progression schedule. Based on typical recommendations for novice kettlebell training, women started with an $8 \mathrm{~kg}$ kettlebell and men with $12 \mathrm{~kg}$. Commercially available kettlebells are divided by $4 \mathrm{~kg}$ increments $(4 \mathrm{~kg}-8 \mathrm{~kg}-12 \mathrm{~kg}-16 \mathrm{~kg}$ - $20 \mathrm{~kg}$ and so on). This implies that a more specific percentage of the maximal load that can be lifted only once (1 repetition maximum or $1 \mathrm{RM})$ is not practical. In addition, the ballistic nature of the kettlebell swing is not suited for 1RM testing.

As participants progressed through the intervention, they had the option to choose a heavier weight when they could complete all ten intervals with the most difficult progression (ie, the one-handed swing). 
Adherence, dropouts, and adverse events

Participants of the training group participated on average 2.1 times per week $(\mathrm{N}=20)$ corresponding to an adherence of $70 \%$. The median number of training sessions during the 8 weeks was $18.5\left(25^{\text {th }}\right.$ percentile 16.8 and $75^{\text {th }}$ percentile 19.3). One participant of the training group dropped out during the initial week of training with no reason given, another due severe neck pain that lasted a week, and a third participant due to long-term sickness absence not related to the training. The latter two of these participants volunteered to reply to the follow-up questionnaire, and we thus included them in the intention-to-treat analysis. No long-lasting or major complications occurred due to the training.

In the control group, one participant did not reply to follow-up questionnaire and two did not show up for the follow-up examination.

\section{Exercise progression}

For the two-handed kettlebell swing, the average kettlebell weight and number of sets performed during the initial (weeks 1-2) and final (weeks 7-8) weeks of training was $8.3 \mathrm{~kg}$ and 23.2 sets and $12.4 \mathrm{~kg}$ and 22.1 sets, respectively. For the one-handed kettlebell swing, the average kettlebell weight and number of sets performed during the initial and final two weeks of training was 8.1 $\mathrm{kg}$ and 27.2 sets and $12.0 \mathrm{~kg}$ and 15.8 sets, respectively. During weeks $1-4$, the work-to-rest ratio was 1:2 (30 seconds of work followed by 60 seconds of rest). The rest time was reduced from the beginning of week 5 and maintained at 30 seconds for the remaining intervention period (ie, a work-to-rest ratio of 1:1).

\section{Co-interventions}

Participants in both groups received the recommendation to continue their usual physical activities alongside the intervention. The participants' level of leisure-time physical activity, registered with a modified version of the Saltin \& Grimby questionnaire (20), showed a slight increase from March-May 2010 in both groups but no significant difference between groups.

\section{Statistical analysis}

We performed all statistical analyses in accordance with the intention-to-treat principle. Between-group differences for the change from baseline to follow-up were determined by analysis of variance using the mixed procedure of the SAS statistical software, version 9.2 (SAS institute, Cary, NC, USA).

A priori power analysis based on previous measurements showed that 16 participants of each group was sufficient to test the null-hypothesis of equality (minimal relevant difference of pain intensity $=1, \beta=0.80$, $\alpha=0.05)$ (11).

\section{Results}

Figure 3 shows the change in neck/shoulder and lowback pain from baseline to follow-up. A priori hypothesis testing of main effects showed a significant group by time interaction for pain intensity of the neck/shoulder $(\mathrm{P}=0.02)$, pain intensity of the low back $(\mathrm{P}=0.05)$, and muscle strength of the back extensors $(\mathrm{P}=0.0005)$, with the training group improving significantly more than the control group (table 2). There was no significant group

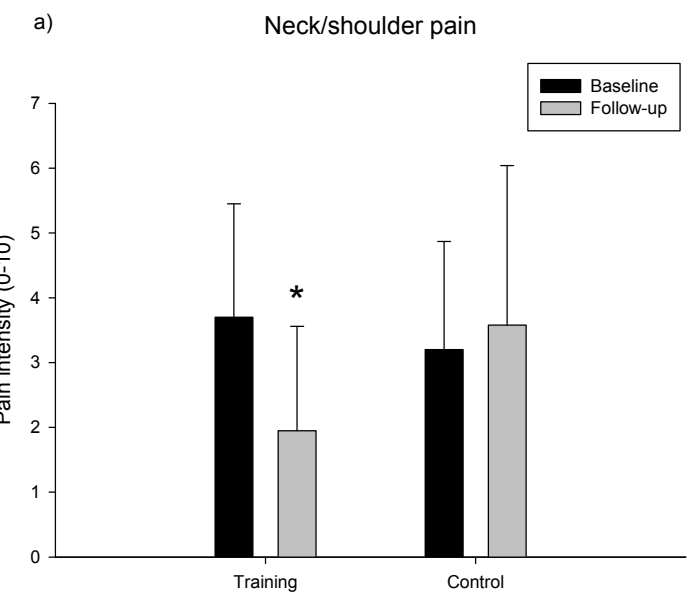

b) Low-back pain

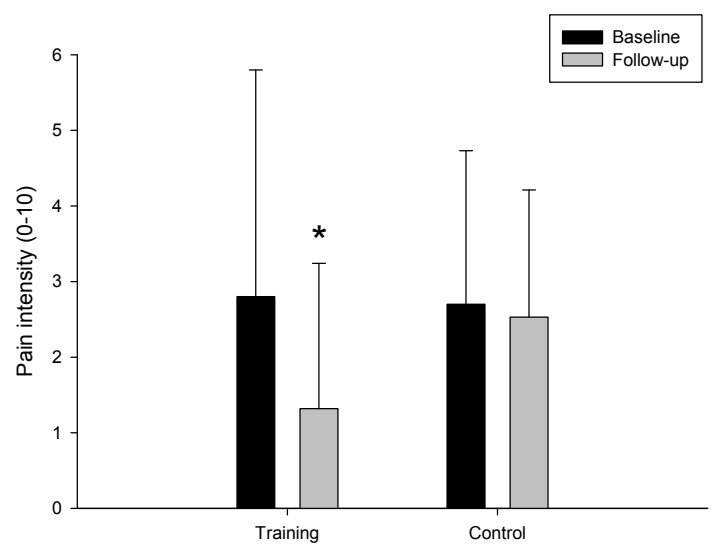

Figure 3. Pain intensity of the neck/shoulder (a) and low back (b) at baseline and 8-week follow-up in the training and control group. Compared with the control group, pain decreased significantly in the training group in both the neck/shoulder and low-back region. ${ }^{*} \mathrm{P}<0.05$. Values are means (standard deviation). 
Table 2. Changes in intensity of pain, muscle strength, and fitness at 8-week follow-up. Differences for each group separately are shown on the left, and contrasts between the groups on the right. [ $95 \% \mathrm{Cl}=95 \%$ confidence interval; MVC=maximal voluntary contraction (ie, muscle strength); $\mathrm{VO}_{2} \mathrm{Max}=$ maximal oxygen uptake.]

\begin{tabular}{|c|c|c|c|c|c|c|c|}
\hline \multirow[t]{3}{*}{ Outcome measure } & \multicolumn{6}{|c|}{ Difference from baseline to follow-up } & \multirow[t]{3}{*}{ P-value } \\
\hline & \multicolumn{2}{|c|}{ Training } & \multicolumn{2}{|c|}{ Control } & \multicolumn{2}{|c|}{ Training versus control } & \\
\hline & Mean & $95 \% \mathrm{Cl}$ & Mean & $95 \% \mathrm{Cl}$ & Mean & $95 \% \mathrm{Cl}$ & \\
\hline \multicolumn{8}{|l|}{ Intensity of pain $(0-10)$} \\
\hline Neck/shoulder & -1.7 & $-2.9--0.5$ & 0.3 & $-0.9-1.5$ & -2.1 & $-3.7--0.4$ & 0.02 \\
\hline Low back & -1.6 & $-2.5--0.6$ & -0.2 & $-1.2-0.7$ & -1.4 & $-2.7--0.02$ & 0.05 \\
\hline \multicolumn{8}{|l|}{ MVC (Nm) } \\
\hline Back extension & 19.6 & $12.7-26.6$ & 1.3 & $-5.4-8.1$ & 18.3 & $8.6-28.0$ & 0.0005 \\
\hline Trunk flexion & 12.0 & $1.4-22.6$ & 3.7 & $-6.6-14.0$ & 8.3 & $-6.5-23.0$ & 0.26 \\
\hline Shoulder elevation & 7.0 & $2.9-11.0$ & 7.5 & $3.6-11.5$ & -0.6 & $-6.2-5.0$ & 0.84 \\
\hline Aerobic fitness $\left(\mathrm{VO}_{2} \mathrm{Max}: \mathrm{ml} \mathrm{O}_{2} / \mathrm{kg} / \mathrm{min}\right)$ & 2.9 & $0.6-5.3$ & 4.8 & $2.5-7.1$ & -1.8 & $-5.1-1.4$ & 0.26 \\
\hline
\end{tabular}

by time interaction for trunk flexion strength, shoulder elevation strength, and aerobic fitness.

\section{Discussion}

Our randomized controlled trial showed clinically relevant reductions of neck/shoulder and low back pain as well as increased muscle strength of the trunk extensors in response to 8 weeks of ballistic kettlebell training. The training protocol did not significantly improve aerobic fitness or strength of the shoulders and trunk flexors.

This is the first randomized controlled trial investigating the effectiveness of kettlebell training for reducing musculoskeletal pain symptoms. Biomechanically, ballistic kettlebell training involves generation of high peak forces of the posterior muscle chain, mediated by cyclic deceleration-acceleration, in an explosive manner. The highly significant increase in back extensor strength, as opposed to no change in shoulder or trunk flexor strength, validates the specificity of the peak forces generated. This type of ballistic movement is not unlike the explosive pull used by athletes in Olympic-style weightlifting, but is in sharp contrast to the typical advice of slowly controlled exercise for people with musculoskeletal pain symptoms. We utilized the kettlebell swing - both bi- and unilateral - as the main intervention exercise. In short, the kettlebell swing has three main phases: (i) a short initial phase of concentric action, (ii) a coasting phase that relies on the momentum generated in the initial phase, and (iii) a deceleration phase governed by eccentric muscle action. The kettlebell swing is explosive in nature, requiring a rapid force capacity of the working muscles, and imposes a great demand on eccentric muscle action capabilities. Furthermore, the fast rhythmical contraction-relaxation cycles of kettlebell swings are different from other ballistic activities that are a-cyclical and non-repetitive in nature (eg, the Olympic snatch). Our study is the first to demonstrate that ballistic cyclic training with high peak forces markedly lowers pain symptoms in both the neck/shoulder and low-back region. Thus, kettlebell training can be implemented at the workplace to reduce common musculoskeletal pain symptoms.

At the end of the eight-week intervention period, the training group had decreased pain sensation in the low-back and neck/shoulder region by approximately 57\% and 46\%, respectively. Previous strength training studies of similar duration with comparable demographics found moderate $(\sim 17-25 \%)$ decline in non-specific neck/shoulder pain $(21,22)$. A recent study by Andersen and coworkers (11) did, however, show a highly significant decrease $(\sim 79 \%)$ in neck/shoulder pain over the course of a 10 -week $(3 \times 20$ minutes/week $)$ intervention period with heavy (70-80\% 1RM) isolated and controlled resistance training among women with diagnosed trapezius myalgia. In a systematic review, Coury et al (23) reported that six out of eight studies on high-intensity resistance training, as an intervention for low-back pain, had a positive effect on controlling symptoms. However, there are distinct differences in what constitutes "high-intensity" in these studies. For instance, in a study by Maul and coworkers (24) the maximum resistance applied allowed $\geq 15$ repetitions ( $60-65 \% 1 \mathrm{RM}$ ) to be completed, which would be considered as moderate resistance. Contradictory to the general moderately positive outcomes of highintensity resistance training in the treatment of low-back pain, Helmhout et al (25) could not show a significant increase in low-back functioning following a 12-week progressive resistance training protocol specifically targeting the lumbar extensor muscle groups with loads corresponding to approximately $50-70 \%$ of $1 \mathrm{RM}$.

The markedly positive and rapid result on pain in our study compared with previous studies is likely to be 
caused by several factors. In relation to the aforementioned repetitive contraction-relaxation cycle of active muscle during kettlebell swings, functional hyperemia must be considered as a possible contributor to the positive results. Within seconds of initiating dynamic muscular contractions, blood flow increases and vascular resistance decreases due to vasodilatation. This is important due to an increased oxygen demand, subsequent consumption and an increased removal rate of metabolic biproducts. In contrast, prolonged and repetitive low-force muscle contractions in occupational tasks promotes local acidosis, which in turn causes or enhances pain conditions (4) as supported by Andersen and colleagues showing decreased capillarization in type I megafibers (26). When the muscle tissue undergoes rhythmical contractions, the local functional hyperemia mechanisms could normalize intra-muscular metabolite concentrations (eg, reduce acidosis) thereby desensitizing chemonociceptive nerve endings and ultimately reducing pain. Future studies should investigate the mechanisms of pain relief observed in our study.

In contrast to the findings by Farrar et al (15) of a relatively high oxygen uptake during kettlebell swings, we found no difference between the groups for the change in aerobic fitness over the 8-week period. We attribute the lack of change in aerobic fitness to insufficient cardiovascular stimulation in the brief training sessions. The session duration of 20 minutes (consisting of 5-10 minutes of warm-up followed by $10-15$ minutes of kettlebell training) does not leave ample duration to stimulate significant cardiovascular adaptations. Seen in the light of the findings by Farrar et al (15), future studies should investigate the effect on aerobic fitness of more frequent kettlebell training of longer duration per session.

Our study has both strengths and limitations. Its strengths are the examiner-blinded randomized controlled design, the high adherence rate to training, and the small loss of participants to follow-up. The inclusion and exclusion criteria limit the generalizability of our findings to employed adults from occupations with a high prevalence of reported musculoskeletal pain symptoms, but without traumatic injuries or other serious chronic disease. The study's limitations include the relatively small number of participants $(\mathrm{N}=40)$, making detection of small possible differences between the groups difficult.

In conclusion, our randomized controlled trial showed reductions of neck/shoulder and low-back pain as well as increased muscle strength of the trunk extensors in response to 8 weeks of ballistic kettlebell training. However, the training protocol did not significantly improve aerobic fitness or strength of the shoulders and trunk flexors.

\section{Acknowledgements}

The National Research Centre for the Working Environment covered all financial expenses related to the project. Thanks to Jesper Rud Kirkegaard, Pia Synnøve Kreutzfeldt, and Mette Odgaard for their help with the project.

\section{References}

1. Strine TW, Hootman JM. US national prevalence and correlates of low back and neck pain among adults. Arthritis Rheum. 2007;57(4):656-65. doi:10.1002/art.22684.

2. Linton SJ. Risk factors for neck and back pain in a working population in Sweden. Work \& Stress. 1990;4(1):41-9. doi:10.1080/02678379008256963.

3. Andersson GB. Epidemiological features of chronic low-back pain. Lancet. 1999;354(9178):581. doi:10.1016/S01406736(99)01312-4.

4. Larsson B, Sogaard K, Rosendal L. Work related neckshoulder pain: a review on magnitude, risk factors, biochemical characteristics, clinical picture and preventive interventions. Best.Pract.Res.Clin.Rheumatol. 2007;21(3):447-63. doi:10.1016/j.berh.2007.02.015.

5. Cote P, Cassidy JD, Carroll LJ, Kristman V. The annual incidence and course of neck pain in the general population: a population-based cohort study. Pain. 2004;112(3):267-73. doi:10.1016/j.pain.2004.09.004.

6. Juul-Kristensen B, Sogaard K, Stroyer J, Jensen C. Computer users' risk factors for developing shoulder, elbow and back symptoms. Scand J Work Environ Health. 2004;30(5):390-8.

7. Andersen LL, Mortensen OS, Hansen JV, Burr H. A prospective cohort study on severe pain as a risk factor for long-term sickness absence in blue- and white-collar workers. Occup Environ Med. 2010; Nov 11.[Epub ahead of print].

8. Linton SJ, van Tulder MW. Preventive interventions for back and neck pain problems: what is the evidence? Spine. 2001;26(7):778-87. doi:10.1097/00007632-20010401000019 .

9. Burton AK, Balague F, Cardon G, Eriksen HR, Henrotin Y, Lahad A, Leclerc A, Muller G, van der Beek AJ. How to prevent low back pain. Best Pract Res Clin Rheumatol. 2005;19(4):541-55. doi:10.1016/j.berh.2005.03.001.

10. Ylinen J, Takala EP, Nykanen M, Hakkinen A, Malkia E, Pohjolainen T, Karppi SL, Kautiainen H, Airaksinen O. Active neck muscle training in the treatment of chronic neck pain in women: a randomized controlled trial. JAMA. 2003;289(19):2509-16. doi:10.1001/jama.289.19.2509.

11. Andersen LL, Kjaer M, Sogaard K, Hansen L, Kryger AI, Sjogaard G. Effect of two contrasting types of physical exercise on chronic neck muscle pain. Arthritis Rheum. 2008;59(1):84-91. doi:10.1002/art.23256. 
12. Andersen LL, Christensen KB, Holtermann A, Poulsen OM, Sjogaard G, Pedersen MT, Hansen EA. Effect of physical exercise interventions on musculoskeletal pain in all body regions among office workers: A one-year randomized controlled trial. Man Ther. 2010;15(1):100-4. doi:10.1016/j. math.2009.08.004.

13. Andersen LL, Jorgensen MB, Blangsted AK, Pedersen MT, Hansen EA, Sjogaard G. A Randomized Controlled Intervention Trial to Relieve and Prevent Neck/Shoulder Pain. Med Sci Sports Exerc. 2008;40(6):983-90. doi:10.1249/ MSS.0b013e3181676640.

14. Pedersen BK, Saltin B. Evidence for prescribing exercise as therapy in chronic disease. Scand J Med Sci Sports. 2006;16 Suppl 1:3-63. doi:10.1111/j.1600-0838.2006.00520.x.

15. Farrar RE, Mayhew JL, Koch AJ. Oxygen cost of kettlebell swings. J Strength Cond Res. 2010;24(4):1034-6. doi:10.1519/JSC.0b013e3181d15516.

16. Pincus T, Bergman M, Sokka T, Roth J, Swearingen C, Yazici Y. Visual analog scales in formats other than a 10 centimeter horizontal line to assess pain and other clinical data. J Rheumatol. 2008;35(8):1550-8.

17. Kuorinka I, Jonsson B, Kilbom Å, Vinterberg H, BieringSørensen F, Andersson G, Jørgensen K. Standardised Nordic questionnaires for the analysis of musculoskeletal symptoms. Appl Ergo. 1987;18(3):233-7. doi:10.1016/00036870(87)90010-X.

18. Essendrop M, Schibye B, Hansen K. Reliability of isometric muscle strength tests for the trunk, hands and shoulders. Int J Ind Erg. 2001;28(6):379-87. doi:10.1016/S01698141(01)00044-0.

19. Åstrand PO, Rodahl K. Textbook of work physiology: physiological bases of exercise. 3rd edition. New York: McGraw-Hill; 1986.
20. Saltin B, Grimby G. Physiological analysis af middle-aged and old former athletes. Circulation. 1968;38:1104-15.

21. Randlov A, Ostergaard M, Manniche C, Kryger P, Jordan A, Heegaard S, Holm B. Intensive dynamic training for females with chronic neck/shoulder pain. A randomized controlled trial. Clin Rehabil. 1998;12(3):200-10. doi:10.1191/026921598666881319.

22. Hagberg M, Harms-Ringdahl K, Nisell R, Hjelm EW. Rehabilitation of neck-shoulder pain in women industrial workers: a randomized trial comparing isometric shoulder endurance training with isometric shoulder strength training. Arch Phys Med Rehabil. 2000;81(8):1051-8. doi:10.1053/ apmr.2000.7582.

23. Coury HJ, Moreira RF, Dias NB. Evaluation of the effectiveness of workplace exercise in controlling neck, shoulder and low back pain: a systematic review. Rev Bras Fisioter. 2009;13(6):461-79.

24. Maul I, Laubli T, Oliveri M, Krueger H. Long-term effects of supervised physical training in secondary prevention of low back pain. Eur Spine J. 2005;14(6):599-611. doi:10.1007/ s00586-004-0873-3.

25. Helmhout PH, Harts CC, Staal JB, Candel MJ, de Bie RA. Comparison of a high-intensity and a low-intensity lumbar extensor training program as minimal intervention treatment in low back pain: a randomized trial. Eur Spine J. 2004;13(6):537-47. doi:10.1007/s00586-004-0671-y.

26. Andersen LL, Suetta C, Andersen JL, Kjaer M, Sjogaard G. Increased proportion of megafibers in chronically painful muscles. Pain. 2008;139(3):588-93. doi:10.1016/j. pain.2008.06.013.

Received for publication: 16 September 2010 